\title{
A simple method for calculating risks before DNA analysis
}

\author{
MARC JEANPIERRE \\ From Service de Biochimie Génétique et Unité INSERM U129, CHU Cochin, 24 rue du Faubourg Saint- \\ Jacques, 75014 Paris, France.
}

SUMMARY Calculation of carrier risk of an $X$ linked disease may be performed on a small computer after DNA analysis, but a method for rapid hand estimation of the risk is still useful for a quick check of the results and weighing the relative importance of each element of information, such as the determination of a haplotype. Each risk estimation is a function of a prior risk and the product of likelihood ratios and these terms are derived themselves from parameters such as fitness or the relative mutation rate in male and female gametes. Even if it is often difficult to have strong experimental estimation of these variables, the existence of a normal father or grandfather must be considered whenever male fitness is not null. The likelihood ratio for a woman for not being a carrier, when her father is not affected and her mother has herself a likelihood $R$ for not having the mutated gene, may be expressed as the ratio $2 R /\left(C_{m} R+1\right)$, with $C_{m}$ being a function of male fitness and relative mutation rate. $C_{m}$ represents the odds ratio for the mother of a carrier not to be a carrier, given that the father of the known carrier is not affected. This formula can be used recurrently and reduces to $2 R /(R+1)$ in lethal $X$ linked disease. When likelihood ratios are expressed as an algebraic function, maximum values are easily determined, hence fixing the limits of DNA analysis.

In recent years at least four computer programs have been introduced which can provide a genetic risk figure combining pedigree information, carrier detection tests, and DNA analysis. ${ }^{1-4}$ These computations are especially complex when the phase of the marker with the disease gene is unknown or flanking probes are used. Geneticists face one or other situation in most cases of $\mathrm{X}$ linked diseases and computer analysis of the data is the only sensible solution to the problem. However, there is still a requirement for hand calculation of pedigree data before DNA analysis to avoid molecular studies in families where potential carriers have a very low risk, or to determine in others those persons who need further study. Most of the parameters needed for computation are derived from experimental data, and relative mutation rates in male and female gametes, male fitness, and quantitative carrier detection tests are only estimates, so it may be wise to use only simple calculations. There may also be a special role for hand computing as a rough estimation of the risk to check the computed result rapidly: the computer program is assumed to be right but it may be fed

Received for publication 10 August 1987

Revised version accepted for publication 25 November 1987. with incorrect data. This work will be limited to $\mathrm{X}$ linked diseases.

\section{What is the shortest way to the risk figure?}

The risk is often expressed using the odds ratio for the disease, which is more easily comprehended by relatives of the patient. The most simple expression of a Bayesian calculation is:

Posterior odds $=$ (prior odds $) \times($ likelihood ratios $)$.

If we have two or more independent elements of information, the computed likelihood ratio is the product of likelihood ratios for each element. Working with odds ratios has the double advantage of a concise and widely understood figure of risk estimation. ${ }^{6}$ There is, however, a serious drawback in the genetic context: each time we jump from one generation to the next or to the previous one, the probability is simply multiplied by a factor such as 0.5 , while this is not possible for odds ratios. The relation between odds ratios and probability is usually written as:

$$
\text { odds }=\frac{\text { probability }}{1-\text { probability }} \text {. }
$$

With probability below 0.5 a literal use of this 
formula is not convenient since it gives an expression such as $0 \cdot 11: 1$, where $1: 9$ would be expected. It is more simple to write:

$$
\mathrm{d}=\frac{1}{\text { Probability of being a carrier }}=\text { odds for } \text { not being }
$$

If, for example, $p=0 \cdot 027$ then $d=(1 / p)=37$ and the odds are $36: 1$. In the clinical context, any geneticist arriving at a probability figure of 0.027 for being a carrier would be liable to convert it to a ratio of two integers, such as $1 / 37$. If we are dealing with the inverse of the probability we may take advantage of a simple formula expressing the relation between the risk of a daughter $\left(1 / d_{D}\right)$ and the risk of her mother $\left(1 / d_{M}\right)$ :

$$
d_{D}=\left(R_{D}+1\right) \times d_{M} \text {. }
$$

$R_{D}$ is the likehood ratio for the daughter for not being a carrier; the latter relation is just a way of expressing the risk of the daughter as $\operatorname{Pr}$ (daughter is a carrier if mother is a carrier) $\times \operatorname{Pr}($ mother is a carrier), assuming that only one mutation has occurred in the pedigree. The former factor is derived from the relation between odds and probability:

$$
\begin{aligned}
1 / \operatorname{Pr}(\mathrm{D} \text { carrier if } \mathrm{M} \text { carrier }) & =(\text { prior odds }) \times \\
& \text { (likelihood ratio })+1 \\
& =(\text { likelihood ratio })+1 .
\end{aligned}
$$

The existence of a healthy son is an element of information which may be represented by the ratio of two probabilities. Such a likelihood ratio may be defined as being higher than the unit for each situation where the probability of having the mutated gene is lower. The likelihood ratio for a mother of a healthy son is then:

$$
\begin{aligned}
& R=\frac{\text { Probability of having a healthy son if non-carrier }}{\text { Probability of having a healthy son if carrier }} \\
& R=1 / 0 \cdot 5=2 \text {. }
\end{aligned}
$$

One reason for the choice of the likelihood of not being a heterozygote as a computation factor is easier hand calculation (that is, $2^{3} v 0.5^{3}$ ) and another is a convenient scaling of the factors since calculations are always aimed to lower the risk: the odds ratios for being healthy or a non-carrier parallels the likelihood ratios. This choice is arbitrary and not grounded in mathematics as the alternative definition would give the same result with an appropriate formula, since $R$ (carrier) equals $1 / R$ (non-carrier). If necessary, we may compute the probability of being a healthy homozygote from the expression of $\mathrm{d}$ :

$\operatorname{Pr}($ non-carrier $)=1-\frac{1}{d}=\frac{d-1}{d}$.
As a rule, starting from the most proximal known carrier of the mutated gene, either a hemizygote or heterozygote, it is possible to incorporate in the computation all the information available as the product of a prior odds ratio and likelihood ratios, and to derive the risk for the rest of the family with the above formulae.

\section{EXAMPLE}

Fig 1 is an example of a lethal recessive $X$ linked disease, Duchenne muscular dystrophy, where the prior risk in a mother of an isolated case is $2 / 3$, and thus prior odds are $0 \cdot 5: 1$, if we assume in this section that the mutation rate is equal in male and female gametes. In this example, the probability that $\mathrm{A}$ is a heterozygote is $1 / \mathrm{d}$ with $\mathrm{d}$ being $1+0.5 \times R$, with $R$ being the product of the following likelihood ratios:

$\mathrm{R}=2$ for one son.

$R=3$ for a biological test (CK) in the normal range. This figure stems from a two class division of the population: about $33 \%$ of known carriers have a CK value below a limit defined by the normal 95th centile. ${ }^{6}$ Emery had suggested further dividing the normal range of values into more than two discrete classes and assigning to each class the ratio of two probabilities, ${ }^{7}$ the first four likelihood ratios being $5 \cdot 20,4 \cdot 48,2 \cdot 35$, and $0 \cdot 24$. Indeed there is no reason to consider a limited number of classes, since Emery's original data showed that the log of CK fits a normal distribution nicely. ${ }^{6}$ The likelihood ratio for having a CK of any value may be described as a ratio of two probability density functions, such as two Gaussian curves. This avoids a 10 times jump

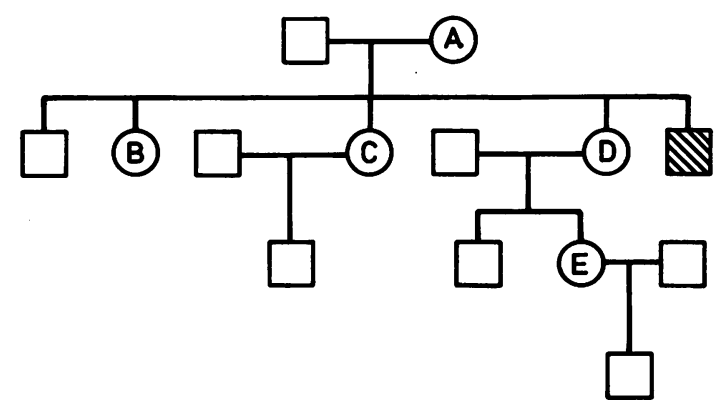

FIG 1 Example of family illustrating the possibility of a rapid hand calculation of the risk of being a carrier. For a daughter with a likelihood ratio for not having the mutated gene $R$, the likelihood factor for her mother is $2 R /(R+1)$. Subjects $A, B, D$ and $E$ have a biological test (here creatine kinase) in the normal range, while the dosage has not been done for daughter $C$. 
when CK rises from $65 \mathrm{IU}$ to $75 \mathrm{IU}$ and such a ratio is already taken into account in some computer programs. ${ }^{1}$ The four parameters are the mean and standard deviation of the two populations (carrier and non-carrier). The existence of a value in the normal range must still be expressed by a ratio of 3 if not obtained with the protocol of the population study, since this method may lead to odd results.

$R=1.5$ for each daughter with a CK in the normal range. The likelihood ratio $R$, derived from the existence of a daughter with a likelihood ratio for being affected $R_{D}$, is:

$$
R=\frac{1}{0.5+0.5 \times R_{D}}=\frac{2 \times R_{D}}{R_{D}+1} .
$$

Since $\mathbf{R}$ is itself a likelihood ratio, recurrent use of this formula is possible in multigeneration families.

$R=1.33$ for each daughter with an unknown $C K$ but who has one normal son: $R=4 /(2+1)$.

$\mathrm{R}=1.82$ for the last daughter of this example, since $\quad R=2 \times(3 \times 2 \times 12 / 7) /(3 \times 2 \times 12 / 7+1)=144 / 79$ (each daughter with a $\mathrm{CK}$ in the normal range and one normal son is represented by a ratio of $2 \times 6 /(6+1)=12 / 7)$.

The final result is thus:

$$
\mathrm{d}_{\mathrm{A}}=1+0.5 \times 2 \times 3 \times 1.5 \times 1.33 \times 1.82=11.9 \text {. }
$$

The probability that $A$ is a carrier is close to $1 / 12$ and the odds for A not being a carrier are 10.9:1. The risk of daughter $\mathrm{D}$ is calculated as follows:

$$
d_{D}=(2 \times 3 \times 1 \cdot 72+1) \times 11 \cdot 9=135 \text {. }
$$

Daughter $D$ has a risk of $1 / 135$ of being a carrier.

Do we have to consider the father?

The notation of this section is derived from the modernisation of the classical usage ${ }^{8}$ introduced by Edwards. ${ }^{9}$ If the $X$ linked disease has a ratio of mutation in male to female gametes of $k$, and the fitness of affected males is $f$, the odds for a mother of an affected child not being a carrier are:

$$
\text { Prior odds }=\frac{1-f}{k+1+f} \text {. }
$$

Table 1 shows the values of this factor as a function of fitness of male hemizygotes and the relative mutation rate. The phenotype of the father of the person seeking advice is usually known and, with this knowledge, the expression of the prior probability is more complex, when the disease is not lethal. We may define one more likelihood ratio $\left(A_{f}\right)$ which may be used together with the general relation.

$$
\begin{aligned}
& \mathrm{A}_{\mathrm{f}}=\frac{1}{\text { Probability that the father of }} \\
& \text { a known carrier is unaffected. }
\end{aligned}
$$

If $q$ stands for the frequency of affected males, $h$ being the proportion of heterozygotes and $u$ the mutation rate at oogenesis, we have:

$$
\begin{aligned}
& A_{f}=\frac{h}{h-f q}=\frac{2 u(k+1+f) /(1-f)}{2 u(k+1+f) /(1-f)-u f(k+2) /(1-f)} \\
& A_{f}=\frac{2(k+1+f)}{k(2-f)+2} .
\end{aligned}
$$

This ratio is 1.00 where fitness is null and 1.64 where fitness $=0.70$ and $k=1.00$ (in Becker type muscular dystrophy, for example), as shown in table 2 .

If one considers now what may be learned from the maternal side, such as healthy brothers or maternal biological tests in the normal range, it is necessary to calculate the probability that the mother of a known carrier is herself a carrier. This figure has been known for more than 50 years to be 0.5 in one population, ${ }^{8}$ but in a family where the father of an obligate carrier is unaffected and $f>0$, a correction must be made.

It is convenient to consider the odds ratio for a

\begin{tabular}{|c|c|c|c|c|c|c|c|}
\hline & $k=1 / 8$ & $k=1 / 4$ & $k=1 / 2$ & $k=1$ & $k=2$ & $k=4$ & $k=8$ \\
\hline$f=0.00$ & 0.89 & 0.80 & 0.67 & $0 \cdot 50$ & 0.33 & $0 \cdot 20$ & 0.11 \\
\hline$f=0 \cdot 10$ & 0.73 & 0.67 & 0.56 & 0.43 & 0.29 & 0.18 & 0.10 \\
\hline$f=0.20$ & 0.60 & 0.55 & 0.47 & 0.36 & 0.25 & 0.15 & 0.09 \\
\hline$f=0.30$ & 0.49 & 0.45 & 0.39 & 0.30 & 0.21 & $0 \cdot 13$ & 0.08 \\
\hline$f=0.40$ & 0.39 & 0.36 & 0.32 & 0.25 & 0.18 & $0 \cdot 11$ & 0.06 \\
\hline$f=0.50$ & 0.31 & 0.29 & 0.25 & 0.20 & 0.14 & 0.09 & 0.05 \\
\hline$f=0.60$ & 0.23 & 0.22 & 0.19 & 0.15 & $0 \cdot 11$ & 0.07 & 0.04 \\
\hline$f=0.70$ & $0 \cdot 16$ & $0 \cdot 15$ & 0.14 & 0.11 & 0.08 & 0.05 & 0.03 \\
\hline$f=0.80$ & $0 \cdot 10$ & $0 \cdot 10$ & 0.09 & 0.07 & 0.05 & 0.03 & 0.02 \\
\hline$f=0.90$ & 0.05 & 0.05 & 0.04 & 0.03 & 0.03 & 0.02 & 0.01 \\
\hline
\end{tabular}
mother of a known carrier not being a carrier, given a normal phenotype of the father of the carrier, and this ratio may be designated $C_{m}$, standing for a maternal carriership constant. If we consider the

TABLE 1 Expression of the odds for the mother of an affected boy not having the mutated gene, as a function of male fitness $(f)$ and the relative mutation rate $(k)$. It is assumed that no other information is available. Example: if $k=1$ and $f=0$, odds are $0.5: 1$, and the probability of being a carrier is $1 /(1+0 \cdot 5)=0.67$. 
TABLE 2 Likelihood ratio for not being a carrier when the phenotype of the father of the person for whom risk is calculated is known to be normal. This ratio is notated $A_{f}$ here.

\begin{tabular}{|c|c|c|c|c|c|c|c|}
\hline & $k=1 / 8$ & $k=1 / 4$ & $k=1 / 2$ & $k=1$ & $k=2$ & $k=4$ & $k=8$ \\
\hline$f=0.00$ & $1 \cdot 00$ & $1 \cdot 00$ & 1.00 & 1.00 & 1.00 & 1.00 & 1.00 \\
\hline$f=0.10$ & 1.09 & 1.09 & 1.08 & 1.08 & 1.07 & 1.06 & 1.06 \\
\hline$f=0.30$ & 1.29 & 1.28 & $1 \cdot 26$ & 1.24 & 1.22 & $1 \cdot 20$ & $1 \cdot 19$ \\
\hline$f=0.40$ & $1 \cdot 39$ & $1 \cdot 38$ & $1 \cdot 36$ & 1.33 & $1 \cdot 31$ & $1 \cdot 29$ & 1.27 \\
\hline$f=0.50$ & 1.49 & 1.47 & 1.45 & 1.43 & 1.40 & $1 \cdot 38$ & $1 \cdot 36$ \\
\hline$f=0.60$ & 1.59 & $1 \cdot 57$ & 1.56 & 1.53 & 1.50 & 1.47 & 1.45 \\
\hline$f=0.80$ & $1 \cdot 79$ & $1 \cdot 78$ & $1 \cdot 77$ & 1.75 & 1.73 & $1 \cdot 71$ & 1.69 \\
\hline$f=0.90$ & 1.89 & 1.89 & $1 \cdot 88$ & 1.87 & $1 \cdot 86$ & $1 \cdot 84$ & 1.83 \\
\hline
\end{tabular}

pedigree in fig 2 , the risk for $\mathrm{B}$ being heterozygous may be computed with the help of the same relation as in the former section:

$$
d_{B}=\left(1+\text { odds } \times R_{B}\right) \times d_{A} .
$$

The odds for a mother of a carrier not being a carrier, given that the father of the carrier is healthy, are:

$$
C_{m}=\frac{u(1+k)}{h / 2}=\frac{(1+k)(1-f)}{k+1+f} .
$$

The likelihood ratio including the information for ascendants on the maternal side not being carriers is then:

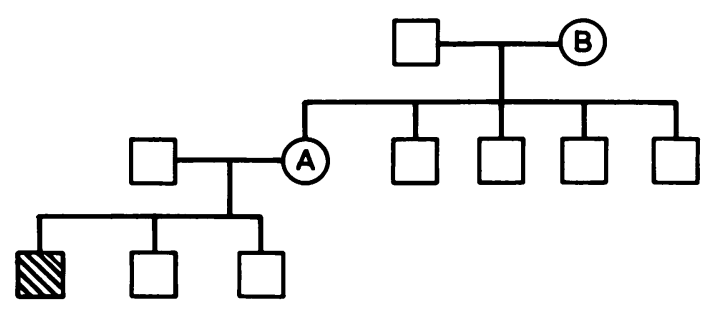

FIG 2 Pedigree of family with an $X$ linked lethal disease.

The risk for $A$ of being a carrier may be computed in one step and the risk for $B$ expressed as a factor of the former result: $d_{A}=1+0.5 \times 4 \times 32 / 17=81 / 17$

$$
d_{B}=81 / 17 \times(16+1)=81 \text {. }
$$

$$
\begin{aligned}
& A_{m}=\frac{1}{1 /\left(\left(C_{m}+1\right) \times R_{m}\right)+\left(1-1 /\left(C_{m}+1\right)\right)} \\
& A_{m}=\frac{\left(C_{m}+1\right) R_{m}}{C_{m} R_{m}+1} .
\end{aligned}
$$

When $f=0, C_{m}$ reduces to 1 and computation of risk from data from the mother or daughters shows obvious similarities, which are exemplified in fig 3 . It may be noted that $A_{f} \times\left(C_{m}+1\right)=2$, since

$A_{f} \times\left(C_{m}+1\right)=\frac{2(k+1+f)}{k(2-f)+2} \times\left(\frac{(1+k)(1-f)}{k+1+f}+1\right)=2$.
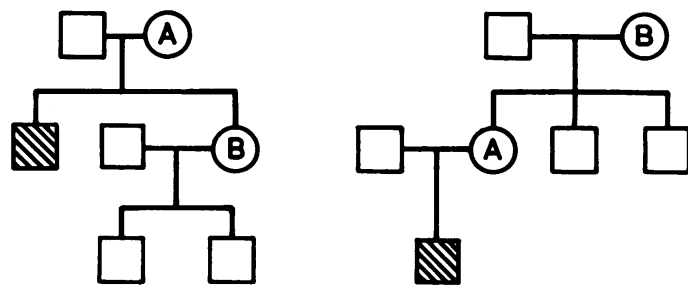

FIG 3 These two hypothetical families illustrate the homology of the expression of the risk for subjects $A$ and $B$. The expression of $d_{B}$ is for both families:

$d_{B}=\left(R_{A}+1\right) R_{B}+1$. This is true only if the male

hemizygotes do not reproduce, otherwise the existence of a normal father of $A$ in the family on the right should be taken

\begin{tabular}{|c|c|c|c|c|c|c|c|}
\hline & $k=1 / 8$ & $k=1 / 4$ & $k=1 / 2$ & $k=1$ & $k=2$ & $k=4$ & $k=8$ \\
\hline$f=0.00$ & 1.00 & $1 \cdot 00$ & 1.00 & 1.00 & 1.00 & 1.00 & 1.00 \\
\hline$f=0 \cdot 10$ & 0.83 & 0.83 & 0.84 & $0 \cdot 86$ & 0.87 & 0.88 & 0.89 \\
\hline$f=0.30$ & 0.55 & 0.56 & 0.58 & 0.61 & 0.64 & 0.66 & 0.68 \\
\hline$f=0.40$ & 0.44 & 0.45 & 0.47 & 0.50 & 0.53 & 0.56 & 0.57 \\
\hline$f=0.50$ & 0.35 & 0.36 & 0.38 & 0.40 & 0.43 & 0.45 & 0.47 \\
\hline$f=0.60$ & 0.26 & $0 \cdot 27$ & 0.29 & $0 \cdot 31$ & $0 \cdot 33$ & $0 \cdot 36$ & 0.38 \\
\hline$f=0.80$ & $0 \cdot 12$ & $0 \cdot 12$ & $0 \cdot 13$ & $0 \cdot 14$ & $0 \cdot 16$ & 0.17 & 0.18 \\
\hline$f=0.90$ & 0.06 & 0.06 & 0.06 & 0.07 & 0.08 & 0.08 & 0.09 \\
\hline
\end{tabular}
into account in the calculation.

TABLE 3 Expression of the odds ratios for the mother of a carrier not being a carrier herself when the father of the carrier is known to have a normal phenotype (otherwise, if the phenotype of the father is unknown, this factor would be 1.00 throughout). This carriership constant is notated $C_{m}$. 
This is not fortuitous since the product can be shown to be the inverse of the probability for a mother of a carrier being a carrier in the population, and this figure is $0 \cdot 5$. The factor $C_{m}$ may be obtained from table 3 or computed from $f$ and $k$. The expression of the two likelihood ratios $A_{f}$ and $A_{m}$ can be condensed to give:

$$
A=\frac{2 R_{m}}{C_{m} R_{m}+1}
$$

When no information can be obtained from maternal ancestors, $R_{m}$ is 1 , and $A$ represents the knowledge of the existence of a normal father:

$$
A=2 /\left(C_{m}+1\right)=A_{f} \text {. }
$$

Fig 4 is an example of a complex pedigree. What are the risks for each woman:

if $\mathrm{f}=0$ and $\mathrm{k}=1$ (that is, DMD),

if $\dot{\mathrm{f}}=\mathbf{0 . 7 0}$ and $\mathrm{k}=4$ (that is, haemophilia $\mathrm{A}$ )?

For $\mathrm{B}$, the calculations are directly computed in one step:

case 1: $d_{B}=1+1 \times 1.78 \times 2 \times 1.60 \times 1.78=11 \cdot 1$

case $2: d_{B}=1+0.26 \times 5.20 \times 2 \times 1.60 \times 1.78=8 \cdot 7$.

In this case, it may be noted that the existence of three normal brothers and a normal father represents a likelihood ratio of $2 \times 2^{3} /\left(0 \cdot 26 \times 2^{3}+1\right)=5 \cdot 20$.

For $\mathrm{A}$, we have:

case 1: $d_{A}=11 \cdot 1 \times\left(2^{3}+1\right)=100$

case 2: $d_{A}=8.7 \times\left(0.26 \times 2^{3}+1\right)=40$.

For $\mathrm{D}$, we have:

case 1: $d_{D}=11 \cdot 1 \times\left(2^{2}+1\right)=55.5$

case $2: d_{D}=8 \cdot 7 \times\left(2^{2}+1\right)=43 \cdot 5$.

In the latter case, it may be noted that the expression of the factor to jump from the risk of a mother to her daughter's risk is independent of the values of $k$ and $f$.

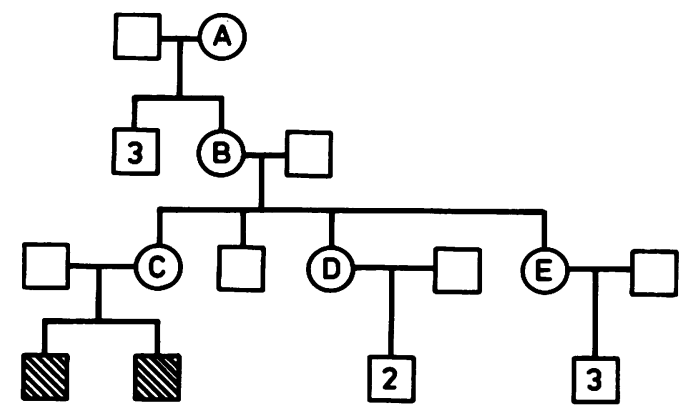

FIG 4 Example of family. illustrating the application of the relations described here. Computations are performed (1) when the disease is lethal and the relative mutation rate in male to female gametes is 1 and (2) when male fitness is 0.70 and the relative mutation rate in gametes is 4 .
How useful are ancestors?

Information from parents may be expressed as a function of the likelihood ratio for the mother of the person for whom risk is calculated. This relation reaches a maximum for $R C_{m}>>1$ and if we designate this limit $\max (\mathrm{A})$, we have the expression:

$$
\max (A)=\frac{2}{C_{m}}=\frac{2(k+1+f)}{(1+k)(1-f)} .
$$

For lethal $\mathrm{X}$ linked diseases $(\mathrm{f}=0)$, information from the maternal side, such as healthy brothers, could lower the estimation of the risk by at most a factor of 2 , while when $f>0$, the maximum weight of information from the parents increases. A high value of $\max (A)$ corresponds to a low frequency of sporadic cases.

How important are uncles of the consultand? If their contribution to the risk of their mother is $R_{u}$, the factor for the risk of their sister is $2 R_{u} /\left(C_{m} R_{u}+1\right)$, while for their niece it is:

$$
R=\frac{2 \times\left(2 R_{u} /\left(C_{m} R_{u}+1\right)\right)}{C_{m} \times 2 R_{u} /\left(C_{m} R_{u}+1\right)+1} .
$$

In order to calculate the maximum influence of uncles, the last relation corresponds to the case where no other information besides the father's phenotype is known. If $C_{m} R_{u}>>1$, we have:

$$
R=\frac{4 / C_{m}}{2+1}=\frac{4}{3 C_{m}}=2 / 3 \max (A) \text {. }
$$

When $f=0$, the existence of healthy uncles and a normal CK test in the grandmother of the person seeking advice may contribute to the risk estimation by a factor of only $1 \cdot 33$.

\section{Germinal mosaicism}

If the possibility of germinal mosaicism is taken into consideration when computing the risk, the basic relation for the risk, written as $1 / p$, of a mother of a carrier being a carrier herself is:

$$
\mathrm{d}=1+\mathrm{L}_{1} \mathrm{R}_{1}+\mathrm{L}_{2} \mathrm{R}_{2}+\mathrm{L}_{3} \mathrm{R}_{3} .
$$

where the $\mathrm{L}_{1}$ variables are the ratios of the prior probability for each hypothesis (non-carrier, maternal mosaicism, and paternal mosaicism) to the prior probability of being a carrier, and the $R_{1}$ variables are the corresponding likelihood ratios. Experimental estimation of prior probabilities and likelihood ratios for a mitotic origin of a mutation is imprecise. This last formula is cumbersome to use and not necessary for most risk estimations. This problem deserves full treatment but even so it should be noted that if germinal mosaicism is not frequent 
(less than $10 \%$ of the non-carriers) and none of the likelihood ratios as computed in the former sections is larger than 20 or so, the ignorance of mosaicism is of little consequence when counselling families before DNA analysis. This is not the case after RFLP studies where the likelihood ratios, that is $(1-\theta) / \theta$, may be very high.

\section{Discussion}

There are two basic strategies for hand calculation of genetic risk: one can calculate the odds for each possible combination of genotypes, usually in a tabular form, ${ }^{9-11}$ or use an algebraic formula. ${ }^{12} 13$ This latter choice could be seen as a set of recipes, but it can be more than this since an algebraic formula can be analysed with the usual mathematical tools and give results such as the notion of a maximum for one component. The strategy for risk calculation developed here stems only from practical considerations: since a risk of 0.045 is expressed as $1 / 22$, why not simply calculate the denominator of a fraction whose numerator is 1 ? If needed, the alternative probability of not being a carrier may be easily computed from $d$ as $\operatorname{Pr}$ (not carrier) $=(d-1) / d$. The concern with easy calculation also resulted in the choice of likelihood ratio of not being a carrier as a way of including pedigree data in the computation. The relative weight of each piece of information may be easily compared: 2 for each healthy son, 1.6 for a daughter with two normal sons, 1.78 for three unaffected brothers. The relations between likelihood ratios of a mother and daugher are easily remembered as they have the same expression when fitness of male hemizygotes is null: $2 R /(R+1)$. Where the mutation is less severe and affected males reproduce, the general expression for the likelihood of being a healthy homozygote when the mother has a likelihood $R$ is $2 R /\left(C_{m} R+1\right)$, given that the father of the consultand is known to have a normal phenotype. The values of $C_{m}$ and the odds for the mother of an isolated case being a healthy homozygote may be either calculated from the estimations of $k$ and $f$, or obtained from tables 1 and 3 . Fitness of male carriers and relative mutation rates may thus be used as parameters, but these variables are obtained from experimental data and may not be known with certainty when the disease is not lethal. Since different mutations lead to distinct phenotypes, neither their probability of occurrence nor the associated fitness are constant features, even if a few mutations are recurrent, as in haemophilia $A .^{14}$ Analysis of tables 1 to 3 shows that the probability of a mother of an isolated case being a carrier is strongly dependent on both parameters, while the probability that a mother of a carrier is herself a carrier, when the father of the carrier is unaffected, is dependent only on fitness. The problem of the relative mutation rate in male and female gametes may thus be ignored whenever the risk is calculated in one branch of a multicase pedigree.

Risk estimations after DNA analysis may not be very different in some families, but this should not prevent us from initiating molecular studies in most cases, though one should bear this fact in mind when requesting blood samples. Molecular analysis of the grandparents of affected children may also lead to fruitful epidemiological studies. ${ }^{15}$

\section{References}

${ }^{1}$ Lathrop GM, Lalouel JM. Easy calculations of lod scores and genetic risks on small computers. Am J Hum Genet 1984; 36:460-5.

2 Sarfarazi M, Williams H. A computer programme for estimation of genetic risk in $\mathrm{X}$ linked disorders, combining pedigree and DNA probe data with other conditional information. $J$ Med Genet 1986;23:40-5.

${ }^{3}$ Clayton JF. A computer programme to calculate risk in $\mathrm{X}$ linked disorders using multiple marker loci. J Med Genet 1986;23:35-9.

4 Andrews DF, Brasher PMA, Manchester KE, et al. DUCHEN: an interactive computer program for calculating heterozygosity (carrier) risks in $\mathrm{X}$-linked recessive lethal diseases, and its application in DMD. Am J Med Genet 1986;25:211-8.

5 Simel DL. Playing the odds. Lancet 1985;i:329-31.

${ }^{6}$ Emery AEH. Carrier detection in sex linked muscular dystrophy. J Genet Hum 1965;14:318-29.

7 Emery AEH. Duchenne muscular dystrophy: genetics aspects, carrier detection and antenatal diagnosis. Br Med Bull 1980;36: $117-22$.

${ }^{8}$ Haldane JBS. The rate of spontaneous mutation of a human gene. J Genet 1935;31:317-26.

9 Edwards JH. The population genetics of Duchenne: natural and artificial selection in Duchenne muscular dystrophy. $J$ Med Genet 1986;23:521-30.

${ }^{10}$ Maag UR, Gold RJM. A simple combinatorial method for calculating genetic risks. Clin Genet 1975;7:361-7.

11 Murphy EA, Mutalik GS. The application of Bayesian methods in genetic counselling. Hum Hered 1969;19:126-51.

12 Morton NE, Chung CS. Formal genetics of muscular dystrophy. Am J Hum Genet 1959;11:360-79.

13 Emery AEH, Holloway S. Use of normal daughters' and sisters' creatine kinase levels in estimating heterozygosity in Duchenne muscular dystrophy. Hum Hered 1977;27:118-26.

14 Youssoufian H, Kazazian HH, Phillips DG, et al. Recurrent mutations in haemophilia A give evidence for $\mathrm{CpG}$ mutation hotspots. Nature 1986;324:380-2.

15 Muller CD, Grimm T. Estimation of the male to female ratio of mutation rates from the segregation of X-chromosomal DNA haplotypes in Duchenne muscular dystrophy families Hum Genet 1986;74:181-3.

Correspondence and requests for reprints to $\mathrm{Dr} M$ Jeanpierre, Service de Biochimie Génétique et Unité INSERM U129, CHU Cochin, 24 rue du Faubourg Saint-Jacques, 75014 Paris, France. 